\title{
Path Tracking Control of Automatic Parking Cloud Model considering the Influence of Time Delay
}

\author{
Yiding Hua, ${ }^{1}$ Haobin Jiang, ${ }^{1,2}$ Yingfeng Cai, ${ }^{2}$ Xupei Zhang, ${ }^{1}$ Shidian Ma, ${ }^{2}$ and Di Wu ${ }^{1}$ \\ ${ }^{1}$ School of Automobile and Traffic Engineering, Jiangsu University, Zhenjiang 212013, China \\ ${ }^{2}$ Automotive Engineering Research Institute, Jiangsu University, Zhenjiang 212013, China \\ Correspondence should be addressed to Haobin Jiang; jianghb@ujs.edu.cn
}

Received 25 October 2016; Revised 6 December 2016; Accepted 19 December 2016; Published 14 February 2017

Academic Editor: Francisco Valero

Copyright (c) 2017 Yiding Hua et al. This is an open access article distributed under the Creative Commons Attribution License, which permits unrestricted use, distribution, and reproduction in any medium, provided the original work is properly cited.

\begin{abstract}
This paper establishes the kinematic model of the automatic parking system and analyzes the kinematic constraints of the vehicle. Furthermore, it solves the problem where the traditional automatic parking system model fails to take into account the time delay. Firstly, based on simulating calculation, the influence of time delay on the dynamic trajectory of a vehicle in the automatic parking system is analyzed under the transverse distance $D_{\text {lateral }}$ between different target spaces. Secondly, on the basis of cloud model, this paper utilizes the tracking control of an intelligent path closer to human intelligent behavior to further study the Cloud Generatorbased parking path tracking control method and construct a vehicle path tracking control model. Moreover, tracking and steering control effects of the model are verified through simulation analysis. Finally, the effectiveness and timeliness of automatic parking controller in the aspect of path tracking are tested through a real vehicle experiment.
\end{abstract}

\section{Introduction}

In recent years, the "parking difficulty" problem in modern cities has become more and more notable. Parking operation in a parking area near crowded and narrow urban roads and community roads is not easy for many drivers $[1,2]$. Therefore, assisted parking driving technology has become one of the research hotspots in the car engineering field and involves knowledge about electromagnetism, environmentaware sensors, signal processing, information fusion, model identification, automatic control and electric power steering direction, and automotive electronics [3-5]. Car makers pay much attention to assisted parking system and successively launched their own assisted parking driving system.

Automatic parking and its control have been studied widely in the domestic and foreign automobile industry, which has yielded fruitful achievements. However, some key technologies in the research of automatic parking have not been researched, such as time delay of automatic parking path tracking and control system.

At present, some research studies have been carried out on the parking path tracking control. Some intelligent control methods are used more extensively, such as fuzzy logic controller, neural network controller, and genetic algorithms (GAs). Yasunobu and Murai [6] proposed a human experience based on fuzzy logic control theory. A fuzzy logic control algorithm was employed to design the parking controller [7] based on a model car test or simulation work. Jenkins and Yuhas [8] introduced a simplified neural network controller trained on the basis of kinematics data. Daxwanger and Schmidt [9] employed fuzzy and neural network schemes to develop a visually based model car backward-parking controller with online autoparking steering angle command. Tayebi and Rachid [10] designed a robust time-varying state feedback parking controller by using the Lyapunov stability rule for a wheel mobile robot. Nevertheless, the fuzzy or neural network controller must be designed on the basis of expertise and trial-and-error work or on a complicated vehicle dynamics model. This is not convenient for practical application. At the same time, neither one of the papers mentioned above addressed the issue about time delay of automatic parking path tracking.

Time delay of automatic parking path tracking mainly includes the following aspects: (1) transmission time delay of measurement signal from the sensor to the control computer; (2) time delay caused by the calculation of control law; (3) 
transmission time delay of control sign from computer to actuator; (4) time delay of actuator; (5) time required to establish control [11]. Time delay greatly affects the performance of the system [12]. It might even cause instability of feedback control system, and so the vehicle will fail to track the ideal parking path. This leads to the failure of the automatic parking system and even collision accidents, which seriously affect the practicability and accuracy of the automatic parking system.

The influence of time delay on the automatic parking system has been reflected in some existing research; for example, Gutjahr and Werling [13] designed time optimal trajectories for braking based on the prediction of the future vehicle motion to compensate for actuator time delay. Choi and Song [14] designed a fault detection and handling method for automatic valet parking to resolve packet loss and time delay of communication. Song et al. [15] designed a lateral controller to be robust enough to compensate for the noise and time delay. However, the uncertainty of time delays was not considered in the papers mentioned above [16-18].

Cloud model is an uncertain transformation model between the qualitative concept proposed by Academician Li Deyi and its quantitative value. Characterized by the coexistence of the uncertainty and certainty as well as the stability during the course of knowledge representation, the cloud model has reflected the fundamental principle of the biological evolution in nature [19-21]. By virtue of its characteristics such as easy implementation of derivation process, simple rules, and strong robustness, it has been widely utilized in the field of intelligent control and subjective evaluation [22]. Normal cloud model is one of the most important among cloud models. Due to its excellent mathematical characteristics, it can describe a large number of uncertain phenomena in different disciplines [23].

Thus, this paper proposes a novel path tracking controller based on cloud model in order to solve the shortcomings of the existing the fuzzy and neural network path tracking controller and to consider the uncertainties of time-delay problems.

The paper is organized as follows. In the next section, a vehicle kinematics model is built and kinematic constraints are analyzed. Then, the influence of time delay on the parking trajectory is simulated with MATLAB. In order to meet the complex control requirements, one-dimensional and twodimensional single rules are organically combined to form the multirule reasoning model. Subsequently, control strategy is verified with straight path and circular path tracking. At last, a real vehicle experiment is presented to verify the effectiveness and timeliness of automatic parking path tracking controller based on cloud model. The paper closes with the conclusions and references.

\section{Analysis of the Vehicle Kinematics Model and Kinematic Constraints}

This paper establishes a kinematic model of vehicles, based on which the path planning method of automatic parking system is analyzed. As shown in Figure $1,\left(x_{r}, y_{r}\right)$ and $\left(x_{f}, y_{f}\right)$ are the

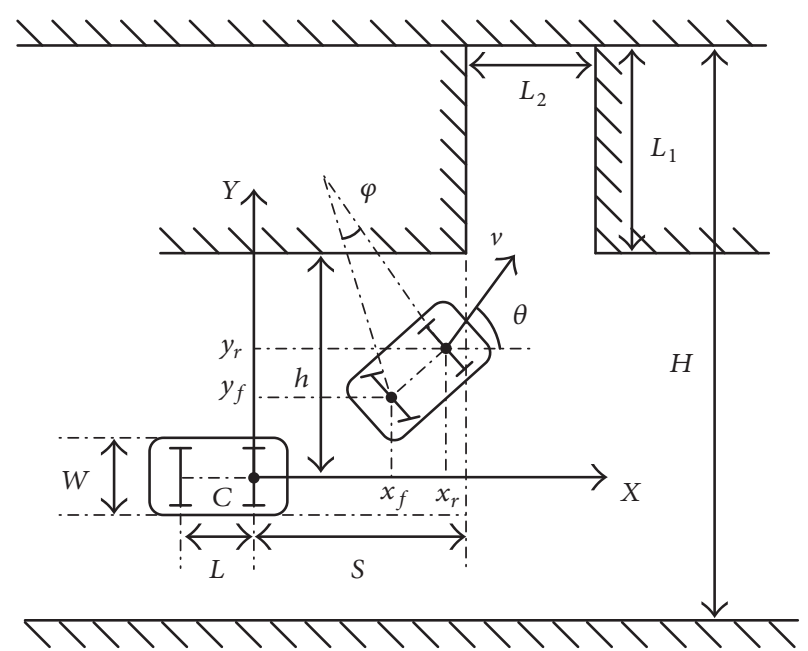

FIGURE 1: Simplified car model for parking.

midpoint coordinates of rear and front axles of the vehicle, respectively, $W$ is indicated as the wheel tread, $H$ is the width of the road, $L_{1}$ and $L_{2}$ are separately the width and length of the target parking space, $h$ is the distance between the midpoint of the rear axle and the lateral barrier, $S$ represents the distance between the midpoint of the rear axle and the tail end of the barrier at the front of the target parking space, $\theta$ denotes the course angle of the vehicle, $\varphi$ is the Ackerman angle, and clockwise direction is positive.

The rear-wheel lateral velocity (vertical to wheel direction) is zero, and the vehicle motion equation in the vertical direction can be obtained:

$$
\dot{x}_{r} \cdot \sin \theta-\dot{y}_{r} \cdot \cos \theta=0 .
$$

According to Ackerman steering geometry principle, Ackerman angle $\varphi$ in the process of vehicle steering approximates to the steering angle of midpoint of vehicle front axle. The central steering angle of the front axle of the vehicle is linearly proportional to steering wheel angle $\gamma$. Hence,

$$
\gamma=K \cdot \varphi
$$

where $K$ is proportional constant.

The midpoint of the rear axle of the parked vehicle is the origin of coordinates, and the coordinate system is established as Figure 1. The positional relationship between the midpoints of front and rear axles of the vehicle can be obtained. Hence,

$$
\begin{aligned}
& x_{r}=x_{f}+L \cdot \cos \theta, \\
& y_{r}=y_{f}+L \cdot \sin \theta .
\end{aligned}
$$

Formula (3) is differentiated to obtain the velocity relationship between the midpoints of front and rear axles of the vehicle. Therefore,

$$
\begin{aligned}
& \dot{x}_{r}=\dot{x}_{f}-L \dot{\theta} \cdot \sin \theta, \\
& \dot{y}_{r}=\dot{y}_{f}+L \dot{\theta} \cdot \cos \theta .
\end{aligned}
$$


Substituting (4) into (1), we can obtain the vehicle kinematics relation:

$$
\dot{x}_{f} \cdot \sin \theta-\dot{y}_{f} \cdot \cos \theta-L \cdot \dot{\theta}=0 .
$$

In addition, at a certain moment of parking in a garage, the velocity of midpoint of the front axle along the direction of axis is as follows:

$$
\begin{aligned}
& \dot{x}_{f}=v \cdot \cos (\theta+\varphi), \\
& \dot{y}_{f}=v \cdot \sin (\theta+\varphi) .
\end{aligned}
$$

Substituting (6) into (5), we can obtain

$$
\dot{\theta}=-\frac{v \cdot \sin \varphi}{L} .
$$

Substituting (6) and (7) into (5), we can obtain the velocity of midpoint of front axle along the direction of axis:

$$
\begin{aligned}
& \dot{x}_{r}=v \cdot \cos \theta \cos \varphi, \\
& \dot{y}_{r}=v \cdot \sin \theta \cos \varphi .
\end{aligned}
$$

Formulas (7) and (8) are denoted as the kinematic equation of vehicle. Hence,

$$
\begin{aligned}
\dot{x}_{r} & =v \cdot \cos \theta \cos \varphi, \\
\dot{y}_{r} & =v \cdot \sin \theta \cos \varphi, \\
\dot{\theta} & =-\frac{v \cdot \sin \varphi}{L} .
\end{aligned}
$$

Formula (9) is utilized to integrate time $t$, and the obtained movement locus equations of midpoint of rear axle are

$$
\begin{aligned}
& x_{r}(t)=-L \cdot \cot \theta \cdot \sin \left(\frac{v \cdot \sin \theta}{L} \cdot t\right) \\
& y_{r}(t)=L \cdot \cot \theta \cdot \sin \left(\frac{v \cdot \sin \theta}{L} \cdot t\right)-L \cdot \cot \theta \\
& x_{r}^{2}+\left(y_{r}+L \cdot \cot \theta\right)^{2}=(L \cdot \cot \theta)^{2}
\end{aligned}
$$

According to the geometric relation between parameters of the vehicle and positions of each coordinate in Figure 1, movement locus equations of four vehicle wheels and envelope points can be obtained. Thereby, the actual movement locus of vehicle in the whole process from starting point to terminal point of parking can be calculated.

Parking movement process should not only meet the vehicle kinematic and geometric characteristics, but also give full consideration to external factors such as security, accuracy, and efficiency of parking. In this section, the influence of barrier in parking environment on the process of parking is analyzed.

Based on the planned parking path, namely, the rear parking locus function, the theoretical curvature $\rho$ of vehicle at arbitrary point in the process of parking in a garage can be given as

$$
\rho=\frac{y^{\prime \prime}}{\left[1+\left(y^{\prime}\right)^{2}\right]^{3 / 2}} .
$$

According to the relation of Ackerman angle,

$$
\tan \varphi=\frac{L}{R},
$$

where $L$ is wheel base, $R$ is the radius of turning circle, and $R=1 / \rho$.

According to formulas (11) and (12), Ackerman angle of the vehicle at arbitrary point is

$$
\varphi=\arctan \left(\frac{L \cdot y^{\prime \prime}}{\left[1+\left(y^{\prime}\right)^{2}\right]^{3 / 2}}\right) .
$$

The movement locus of A, B, C, and D can be obtained based on coordinates of midpoints of rear axle and their mutual relationship. Hence,

$$
\begin{aligned}
& x_{A}=x+\left(L_{f}+L\right) \cdot \cos \theta+\frac{W}{2} \cdot \sin \theta, \\
& y_{A}=y-\left(L_{f}+L\right) \cdot \sin \theta+\frac{W}{2} \cdot \cos \theta, \\
& x_{B}=x+\left(L_{f}+L\right) \cdot \cos \theta-\frac{W}{2} \cdot \sin \theta, \\
& y_{B}=y-\left(L_{f}+L\right) \cdot \sin \theta-\frac{W}{2} \cdot \cos \theta, \\
& x_{C}=x-L_{r} \cdot \cos \theta-\frac{W}{2} \cdot \sin \theta, \\
& y_{C}=y+L_{r} \cdot \sin \theta-\frac{W}{2} \cdot \cos \theta, \\
& x_{D}=x-L_{r} \cdot \cos \theta+\frac{W}{2} \cdot \sin \theta, \\
& y_{D}=y+L_{r} \cdot \sin \theta+\frac{W}{2} \cdot \cos \theta .
\end{aligned}
$$

On the basis of the parking kinematic model established in the above section, there are four positions where the danger exists in the process of parallel parking (as shown in Figure 2): (a) collision between point $\mathrm{C}$ and the left boundary of the carriageway, or the collision between the boundary beyond the current carriageway and vehicle running on the left carriageway in the process of parking; (b) collision between tail of vehicle which has not been parked and the barrier or vehicle at the front of right available parking space; (c) collision between point $\mathrm{B}$ of the vehicle and the barrier after which the vehicle is parked in the space; (d) collision between point $\mathrm{D}$ as well as point $\mathrm{A}$ of the vehicle and the barrier after which the vehicle is parked in the space.

In order to ensure the safety in the parking process and reduce the risk of collision between the vehicle and 


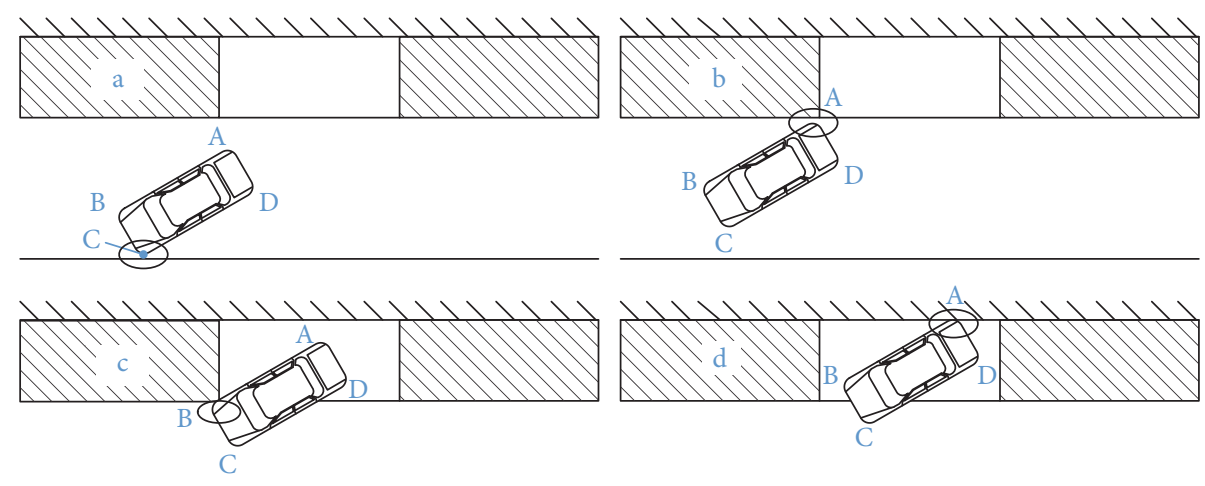

FIGURE 2: Parallel parking possible collisions.

the barrier, locus function needs to meet the following conditions:

$$
\begin{array}{ll}
\text { When } x_{A} \in\left[0, s_{o}-L_{2}\right], & y_{A}<h_{0} ; \\
\text { When } x_{A} \in\left[0, s_{o}-L_{r}\right], & y_{A}<h_{0}+L_{1} ; \\
\text { When } x_{B} \in\left[0, s_{o}-L_{2}\right], & y_{B}<h_{0} ; \\
\text { When } x_{B} \in\left[0, s_{o}-L_{r}\right], & y_{B}<h_{0}+L_{1} ; \\
\text { When } x \in\left[0, s_{o}-L_{r}\right], & y_{C}<h_{0}+L_{1}-h ; \\
\text { When } x=s_{o}-L_{r}, & y_{C}>h_{0} ; \\
\text { When } x=s_{o}-L_{r}, & y_{D}>h_{0} .
\end{array}
$$

The analysis of the constraints of kinematics in the process of parking lays the foundation for vehicle trajectory planning and path tracking in the process of parking and ensures the safety of the running vehicle during parking.

\section{Influence of Time Delay on the Parking Trajectory}

Due to the variation of arc length of vehicle wheels in each process generated from the time delay, this system can calculate the passing arc length of wheels at the three stages of parking on the basis of analysis of the difference in distance $D_{\text {lateral }}$ between the vehicle and target parking space.

In actual parking process, there are time errors in each stage, especially at the points between any two stages. $\Delta L_{1}$, $\Delta L_{2}$, and $\Delta L_{3}$ are assumed as the arc length deviations at each stage under the average velocity speed of $2 \mathrm{~km} / \mathrm{h}$. When $D_{\text {lateral }}$ is $0.5 \mathrm{~m}, 1 \mathrm{~m}$, and $1.5 \mathrm{~m}$, respectively, the influence of time delay on the deviation of parking path is analyzed.

By analyzing Figures 3-5, it can be known that even a short time delay can affect the parking trajectory. The red trajectory in the figure indicates the theoretically ideal parking trajectory obtained from simulation, and the blue trajectory represents the actual parking trajectory affected by time error. The time delay most significantly influences the trajectory between the first and the second stages, and the influence between the second and the third stages is less significant. Furthermore, we can realize that the greater the time delay, the more obvious the trajectory error. If the deviation is excessive, the tested vehicle cannot be accurately parked in the target parking space theoretically. At present, only the influence of the time delay between every two stages on the whole parking trajectory deviation is considered. However, the effect of superposition of time delay on parking trajectory has not been analyzed.

\section{Control Strategy}

This paper constructs the tracking control model and adjusts the characteristic parameters of the cloud on the basis of uncertain cloud reasoning model. The controller can enable the vehicle to rapidly and accurately track the expected path.

4.1. Basic Principles of Cloud Model. The cloud is a model using the linguistic value to represent the uncertainty conversion between a qualitative concept and its quantitative representation. Suppose $U$ is a quantitative domain expressed in precise values and $A$ is a qualitative concept in $U$. If a quantitative value $x \in U$ is a random realization of the qualitative concept $A$ and the membership of $x$ to $A, \mu(x) \epsilon$ $[0,1]$, is a random number with a stable tendency: $\mu: U \rightarrow$ $[0,1], \forall x \in U, x \rightarrow \mu(x)$, then the distribution of $x$ on domain $U$ is called the cloud and each $x$ is called a cloud droplet [24]. Particularly, it is assumed that $R_{1}\left(E_{1}, E_{2}\right)$ can be expressed as a random function obeying normal distribution, where $E_{1}$ is expected value and $E_{2}$ is standard deviation. If $x(x \in U)$ and $\mu(x)$ satisfy the equations, which can be expressed as

$$
\begin{aligned}
& x=R\left(E_{x}, E_{n}\right), \\
& p=R\left(E_{n}, H_{e}\right), \\
& \mu=e^{-(1 / 2)\left(\left(x-E_{x}\right) / p\right)^{2}},
\end{aligned}
$$

then the distribution of $x$ on domain $U$ is called the normal cloud [25]. $\mu$ is the degree of membership cloud for input variable $x$. Cloud numerical features can be utilized to reflect the overall characteristics of the qualitative concept expressed 


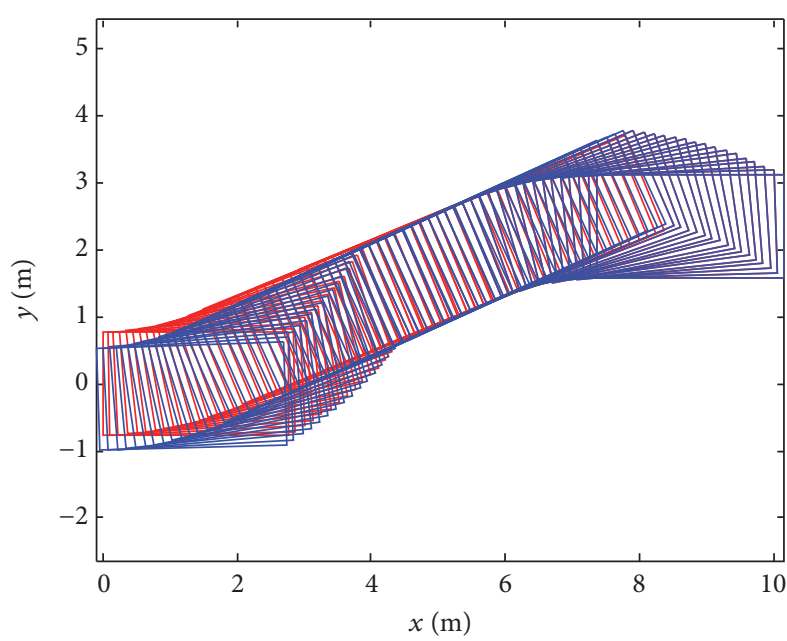

(a)

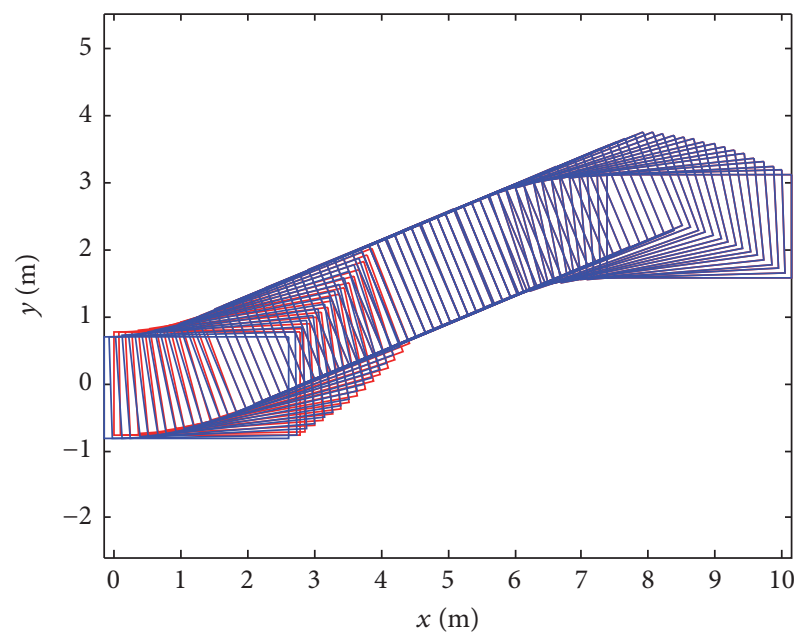

(c)

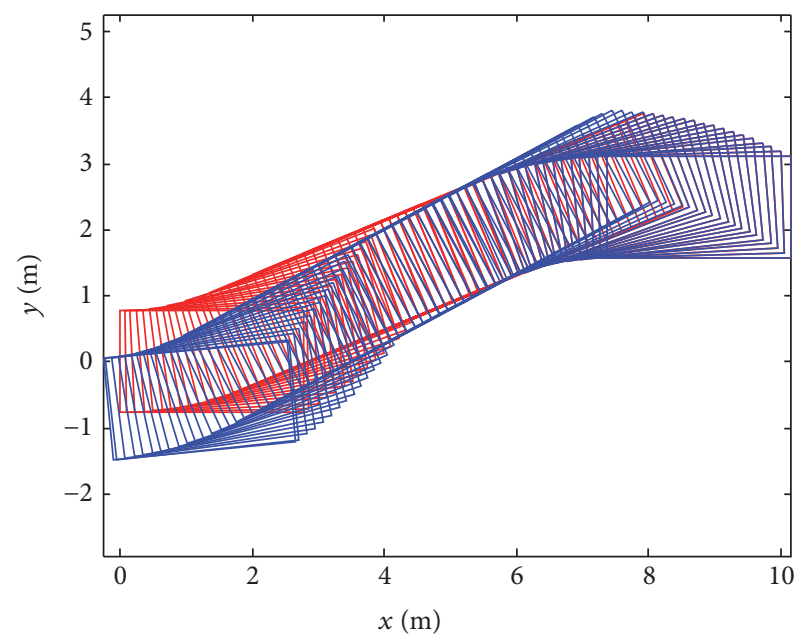

(b)

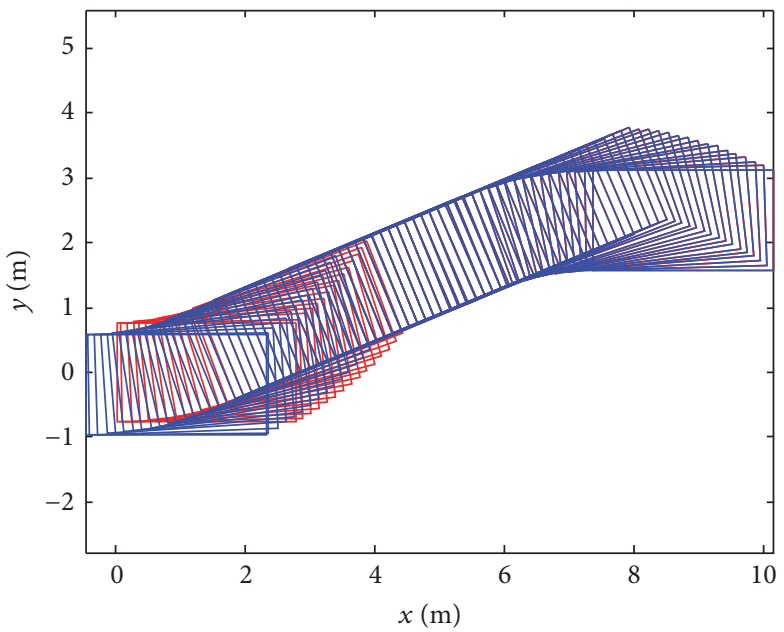

(d)

FiguRE 3: Effect of time delay on parking trajectory when $D_{\text {lateral }}=0.5 \mathrm{~m}$. (a) The time delay is $0.3 \mathrm{~s}$ between the first and the second stages. (b) The time delay is $0.9 \mathrm{~s}$ between the first and the second stages. (c) The time delay is $0.3 \mathrm{~s}$ between the second and the third stages. (d) The time delay is $0.9 \mathrm{~s}$ between the second and the third stages.

by the cloud model. The cloud droplets have three numerical features. In (16), $E_{x}, E_{n}$, and $H_{e}$ denote the expectation, entropy, and hyperentropy, respectively, which are used to describe the numerical characteristics of cloud. $E_{x}$ is the expectation of cloud droplets in the distribution of the domain and is the most typical point that represents this qualitative concept. $E_{n}$ is the uncertain measurement of the qualitative concept and reflects the relevance of fuzziness and randomness. $H_{e}$ is the uncertain measurement of entropy and is determined by the fuzziness and randomness.

$R_{2}$ is assumed to be a two-dimensional random function obeying normal distribution, where $E_{1}$ and $E_{2}$ are expected value and $E_{3}$ and $E_{4}$ are standard deviation. Hence,

$$
\begin{aligned}
(x, y) & =R_{2}\left(E_{1}, E_{2}, E_{3}, E_{4}\right), \\
\left(p_{x}, p_{y}\right) & =R_{2}\left(E_{3}, E_{4}, H_{e_{x}}, H_{e_{y}}\right), \\
\mu^{\prime} & =e^{-(1 / 2)\left[\left(x-E_{1}\right)^{2} / p^{2}{ }_{x}+\left(\left(y-E_{2}\right)^{2} / p^{2}{ }_{y}\right)\right]} .
\end{aligned}
$$

The cloud model constructed by data meeting formula (17) on $\operatorname{drop}\left(x, y, \mu^{\prime}\right)$ is a two-dimensional normal cloud model, which is abbreviated as two-dimensional normal cloud. The data constructing this could model for $\operatorname{drop}\left(x, y, \mu^{\prime}\right)$ is called two-dimensional cloud droplets [26].

Three normal clouds with different characteristics are shown in Figure 6. Compared with the three clouds, it can be found that the smaller the value of $E_{n}$ is, the more divergent the cloud will be. And it can also be found that the bigger the value of $H_{e}$ is, the more divergent the cloud will be. Normal cloud is composed of some cloud droplets, which can reflect the fuzziness. Cloud model is not described through certain functions, therefore, to enhance the processing capacity for uncertainty.

The process of reasoning about the uncertain rules of the cloud model is the target rules inferred and calculated through the known conditions in a certain environment [27]. Cloud Generator (CG) is the algorithm of cloud model. The 


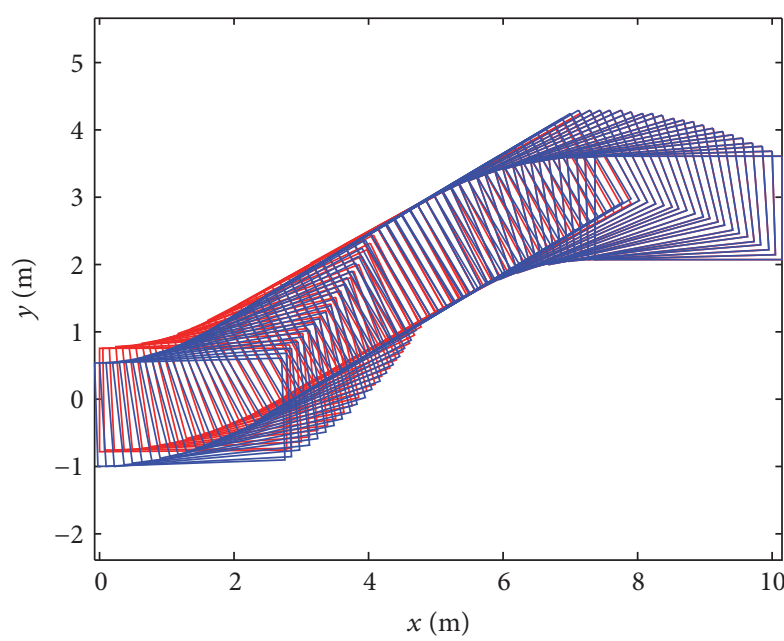

(a)

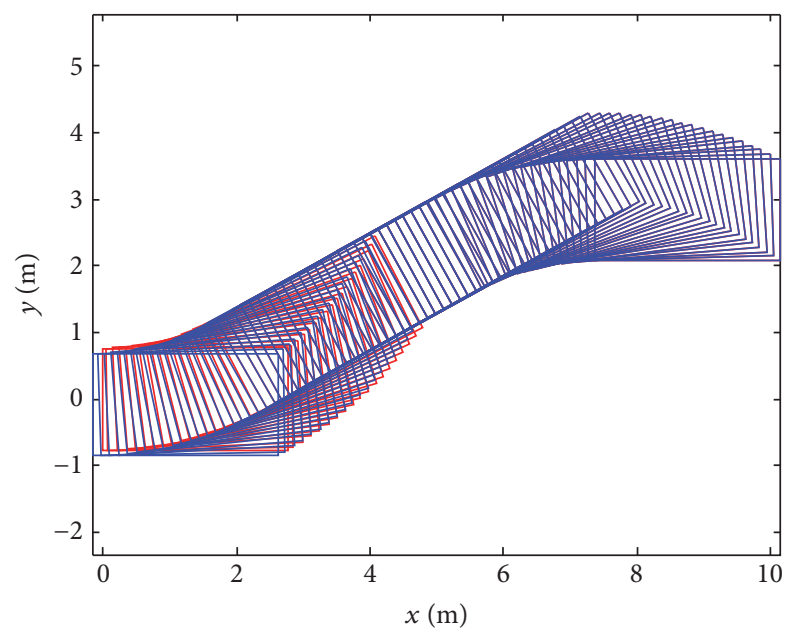

(c)

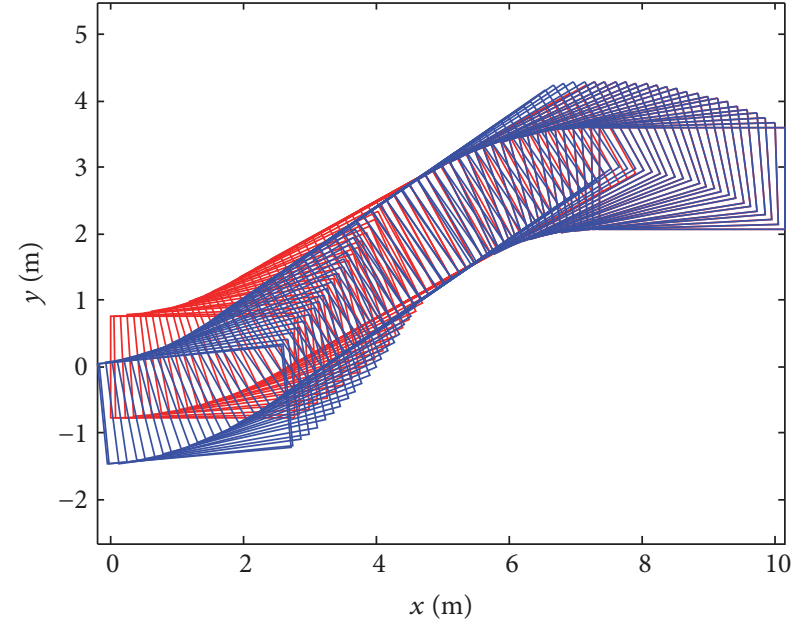

(b)

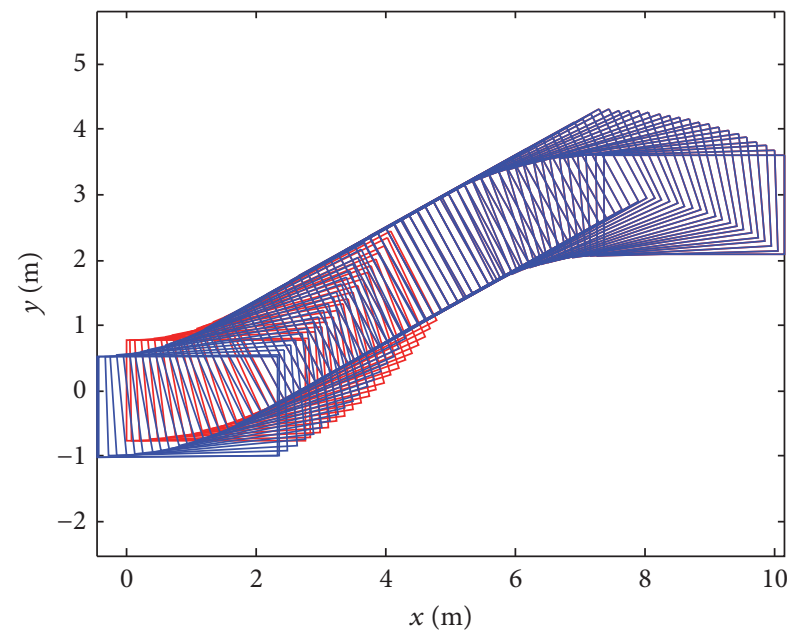

(d)

Figure 4: Effect of time delay on parking trajectory when $D_{\text {lateral }}=1.0 \mathrm{~m}$. (a) The time delay is $0.3 \mathrm{~s}$ between the first and the second stages. (b) The time delay is $0.9 \mathrm{~s}$ between the first and the second stages. (c) The time delay is $0.3 \mathrm{~s}$ between the second and the third stages. (d) The time delay is $0.9 \mathrm{~s}$ between the second and the third stages.

inputs of the generator are the three numerical characteristics. The outputs are cloud droplets. CG can realize the mapping from qualitative data to quantitative data. There are many CGs such as Forward Cloud Generator, Backward Cloud Generator, $X$ Condition Cloud Generator, and $Y$ Condition Cloud Generator.

Consider the one-dimensional cloud model of single-rule reasoning: if $x$, then $y$. Its structure is shown in Figure 7(a), where $x$ condition cloud model is $\left(E_{x}, E_{n x}, H_{e x}\right)$ and $y$ condition cloud model is $\left(E_{y}, E_{n y}, H_{e y}\right)$. When the rule is activated more than once by the quantitative input value $x$, $\mathrm{CG}_{X}$ produces a set of $\mu_{i}$ values, while $\mu_{i}$ generates a set of $y_{i}$ values through $\mathrm{CG}_{Y} \cdot \mathrm{CG}_{X}$ can be presented by formula (16). $\mathrm{CG}_{Y}$ can be expressed as follows:

$$
\begin{aligned}
& P=R\left(E_{n y}, H_{e y}\right) \\
& y_{i}=E_{y} \pm \sqrt{-2 \ln \left(\mu_{i}\right)} \cdot P .
\end{aligned}
$$

Both $X$ and $Y$ CGs are the basis of the construction of cloud model uncertainty reasoning, which form a single-rule generator after being connected. The two-dimensional cloud model of single-rule reasoning is shown in Figure 7(b).

4.2. Path Tracking Control Model. The basic idea of the parking path tracking control model is as follows: the vehicle runs at a certain speed. According to the known path and the running parameters of the vehicle, proper steering angle control parameters are calculated. The driving direction of the vehicle is changed, and it runs along the preplanned path. In this process, the steering control parameters are obtained through relevant inference based on the given running parameters. The mapping relation of individual one-dimensional or two-dimensional single-rule reasoning between input and output is simple and can hardly meet the complex control requirements. In this paper, the abovementioned one-dimensional and two-dimensional single 


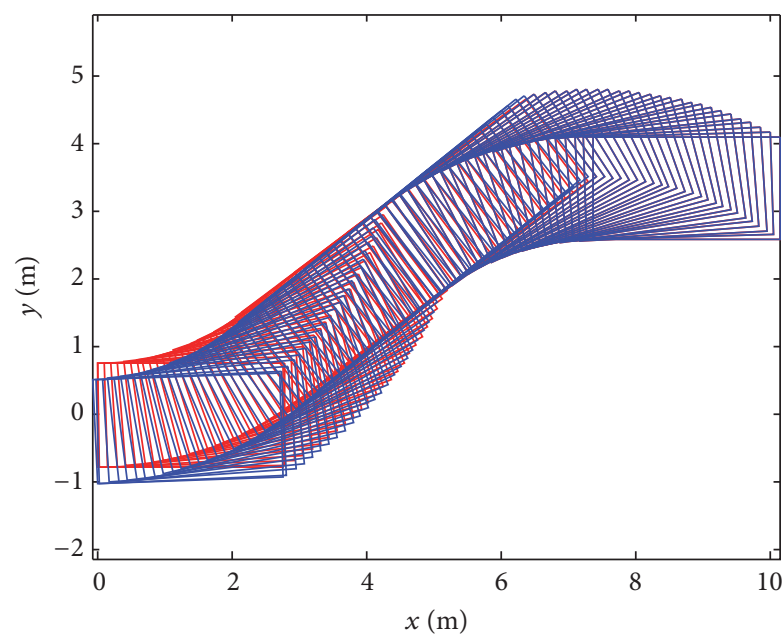

(a)

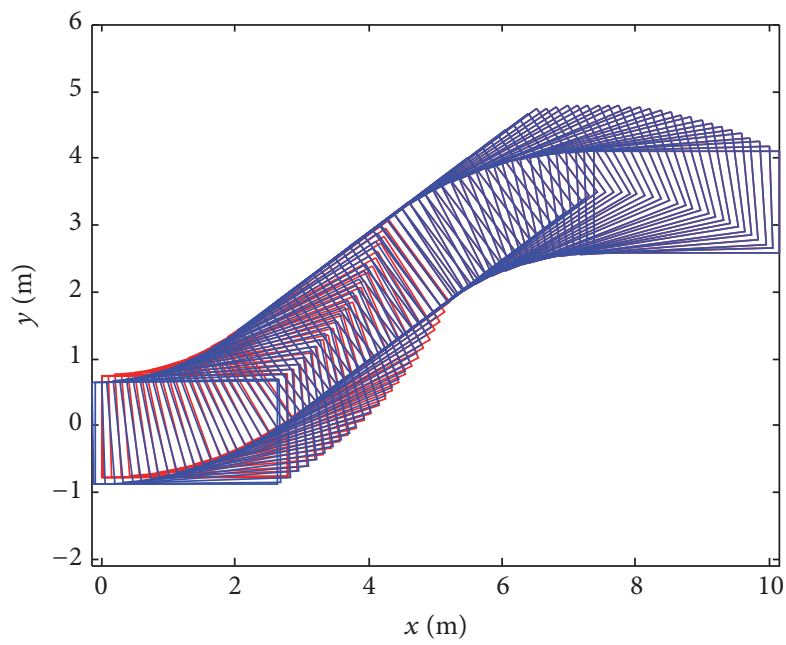

(c)

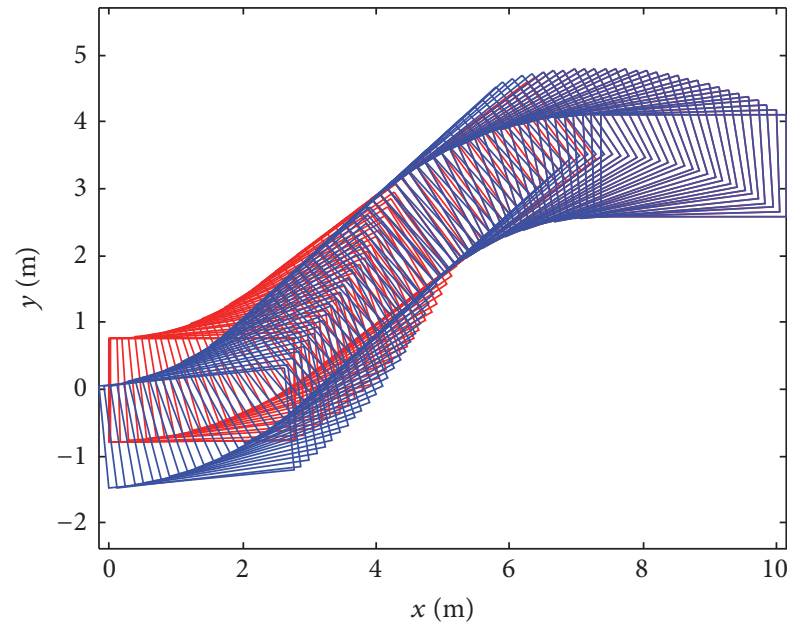

(b)

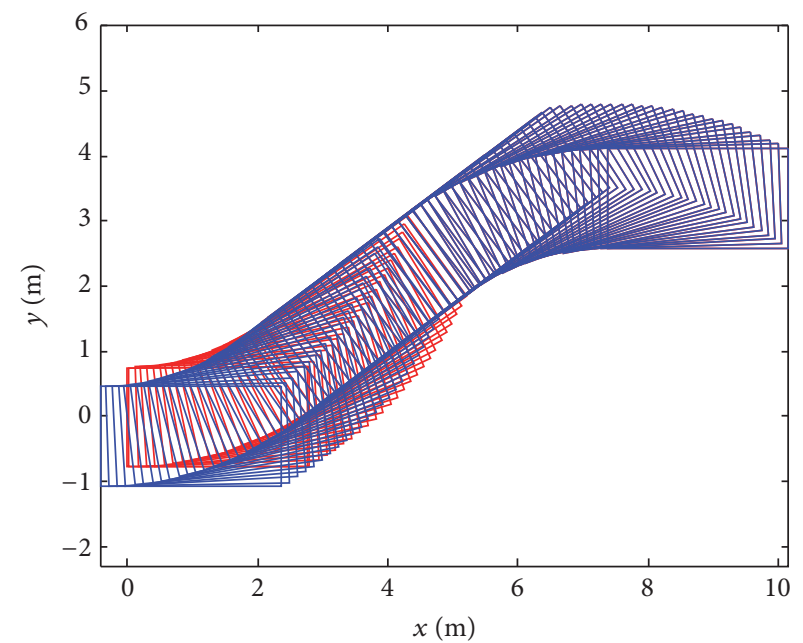

(d)

FIGURE 5: Effect of time delay on parking trajectory when $D_{\text {lateral }}=1.5 \mathrm{~m}$. (a) The time delay is $0.3 \mathrm{~s}$ between the first and the second stages. (b) The time delay is $0.9 \mathrm{~s}$ between the first and the second stages. (c) The time delay is $0.3 \mathrm{~s}$ between the second and the third stages. (d) The time delay is $0.9 \mathrm{~s}$ between the second and the third stages.

rules are organically combined to form the multirule reasoning model shown in Figure 8. This model can complete both linear and nonlinear reasoning requirements. A more difficult prediction at a higher level can be carried out by multirule deduction.

PID controller has the advantages of simple structure, convenient adjustment, and parameter tuning contact in engineering, but the choice of traditional PID regulator parameters mainly depends on repeated tests and experience, when the state of the object changes. PID controller consists of the proportion of unit $\mathrm{P}$, integral unit $\mathrm{I}$, and differential unit D. In this paper, in order to facilitate the comparison, the PID controller is designed. PID controller of automatic parking is applied to adjust actual steering wheel angle output by setting $k_{p}, k_{i}$, and $k_{d}$.

The working process of the model is as follows: the controlled quantity relevant parameters $x_{1}, x_{2}$, and $x_{3}$ are assumed as the input of the multirule generator, according to which the multirule derivation is carried out. The results are applied to the adjustment of constant coefficients $k_{\mathrm{PD}}$ and $k_{\mathrm{I}}$ so as to adjust and control the output parameter range. Finally, the weighted average output steering electrical machine is controlled in real time.

Input variables $x_{1}, x_{2}$, and $x_{3}$ activate different $X$ conditional cloud $\mathrm{PCG}_{A i, B i}$ and $\mathrm{CG}_{a j}$, generating different $\mu_{i}$ and $\mu_{j}$, respectively. The cloud droplet is generated through $Y$ conditional cloud $\mathrm{CG}_{c i}$ and $\mathrm{CG}_{b j}$. Through the weighted average after adjustment of constant coefficient, the inferential results could be obtained, and the generator process is completed.

Inference rule of rule base of multidimensional cloud model is formulated.

If $A_{i}, B_{j}$, and $a_{k}$, then $R_{i j k}$, where the value ranges of $i, j$, and $k$ are $1 \sim 5$. The corresponding rules of the front and rear part are constructed in Figure 9. The consequents of the rules library are listed in Table 1. 
TABLE 1: The consequents of the rules library.

\begin{tabular}{|c|c|c|c|c|}
\hline$R_{11 k}=\left(C_{1}, b_{1}\right)$ & $R_{12 k}=\left(C_{1}, b_{k}\right)$ & $R_{13 k}=\left(C_{1}, b_{k}\right)$ & $R_{14 k}=\left(C_{2}, b_{k}\right)$ & $R_{15 k}=\left(C_{2}, b_{k}\right)$ \\
\hline$R_{21 k}=\left(C_{1}, b_{k}\right)$ & $R_{22 k}=\left(C_{1}, b_{k}\right)$ & $R_{23 k}=\left(C_{2}, b_{k}\right)$ & $R_{24 k}=\left(C_{3}, b_{k}\right)$ & $R_{25 k}=\left(C_{3}, b_{k}\right)$ \\
\hline$R_{31 k}=\left(C_{2}, b_{k}\right)$ & $R_{32 k}=\left(C_{2}, b_{k}\right)$ & $R_{33 k}=\left(C_{3}, b_{k}\right)$ & $R_{34 k}=\left(C_{4}, b_{k}\right)$ & $R_{35 k}=\left(C_{4}, b_{k}\right)$ \\
\hline$R_{41 k}=\left(C_{3}, b_{k}\right)$ & $R_{42 k}=\left(C_{4}, b_{k}\right)$ & $R_{43 k}=\left(C_{4}, b_{k}\right)$ & $R_{44 k}=\left(C_{5}, b_{k}\right)$ & $R_{45 k}=\left(C_{5}, b_{k}\right)$ \\
\hline$R_{51 k}=\left(C_{4}, b_{k}\right)$ & $R_{52 k}=\left(C_{5}, b_{k}\right)$ & $R_{53 k}=\left(C_{5}, b_{k}\right)$ & $R_{54 k}=\left(C_{5}, b_{k}\right)$ & $R_{55 k}=\left(C_{5}, b_{k}\right)$ \\
\hline
\end{tabular}
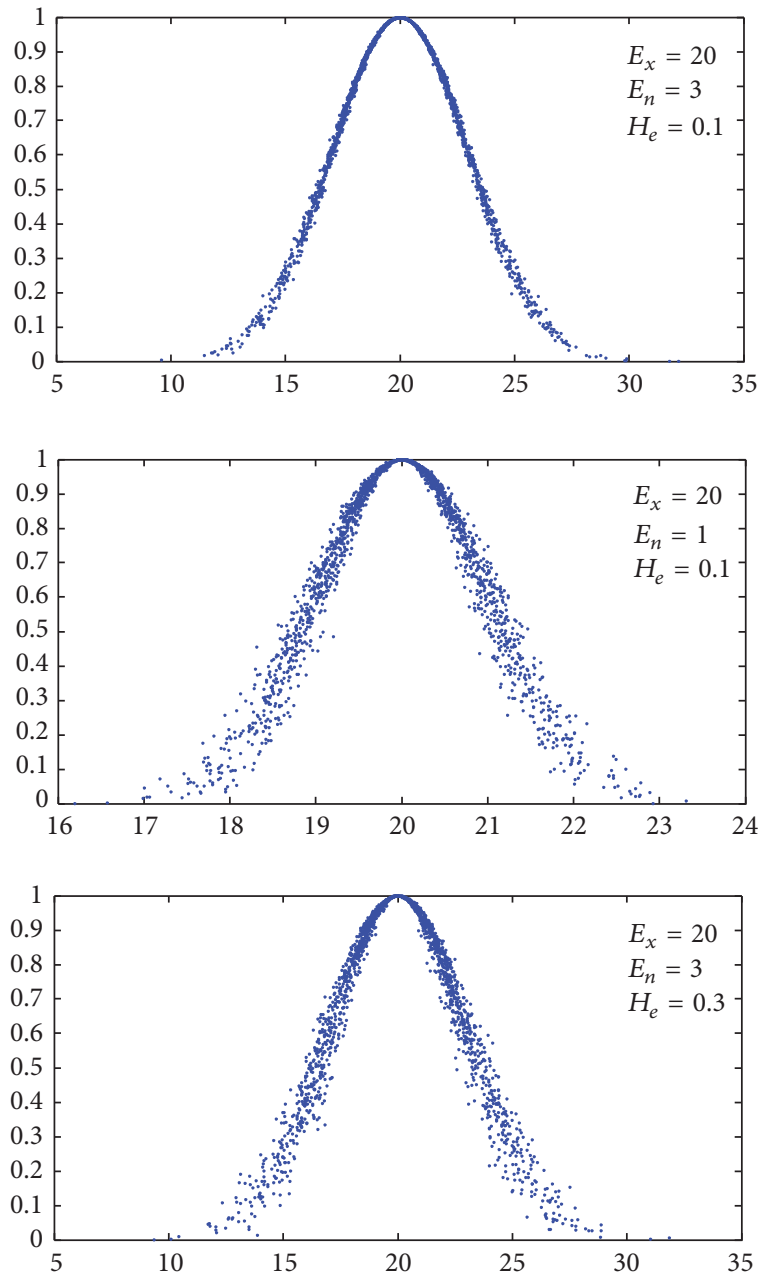

FIgURE 6: Three examples of the normal cloud.

$A_{i}$ means deviation of actual steering wheel angle and theoretical steering wheel, $B_{j}$ means deviation differential of actual steering wheel angle and theoretical steering wheel, $a_{k}$ means deviation integral of actual steering wheel angle and theoretical steering wheel, $C_{i}$ means control outputs of PD type two-dimensional cloud model mapper, and $b_{j}$ means control outputs of I type two-dimensional cloud model mapper.

According to the contents discussed above, it is known that if parking path is given, Ackerman angle of the vehicle at arbitrary point on the path could be obtained. Parking kinematic model is taken as the controlled object of trajectory tracking control model. In order to control the vehicle tracking path through control model, the deviation $\mu$ between Ackerman angle $\varphi$ of the vehicle and the theoretical value $\varphi_{0}$ is taken as the input of the control model, among which $\varphi_{0}$ can be calculated through formula (13). By inputting the deviation $\mu$, deviation integral $\mu_{i}$, and deviation differential $\mu_{c}$ into the cloud model generator, the output controlled variable can be rapidly adjusted based on variation of deviation, so that the vehicle can be driven tracking the path.

In actual operation, in order to facilitate the measurement, the variable input of the control model is converted into angle deviation value of steering wheel according to formula (13). To avoid the adverse effects of three aspects of time delay, that is, (1) time delay in calculation of steering control law, (2) transmission time delay of control sign from microprocessor to steering motor, and (3) time required to establish control algorithm, the range of deviation $\mu$ is selected as $(-10,10) .-10$, $-5,0,5$, and 10 are, respectively, taken as the five expected values of the front five rules. The numerical characteristic value of cloud is input. The algorithm can reduce the negative influence of time delay on the basis of guaranteeing higher control precision. Hence,

$$
\begin{aligned}
& A_{1}=B_{1}=a_{1}=(-10,5,0.1) ; \\
& A_{2}=B_{2}=a_{2}=(-5,3,0.1) ; \\
& A_{3}=B_{3}=a_{3}=(0,3,0.1) ; \\
& A_{4}=B_{4}=a_{4}=(5,3,0.1) ; \\
& A_{5}=B_{5}=a_{5}=(10,5,0.1) .
\end{aligned}
$$

Similarly, the output of the control model is the adjustment amount of the steering angle, and the numerical characteristic value is output. Therefore,

$$
\begin{aligned}
& C_{1}=b_{1}=(-12,5,0.1) ; \\
& C_{2}=b_{2}=(-6,3,0.1) ; \\
& C_{3}=b_{3}=(0,3,0.1) ; \\
& C_{4}=b_{4}=(6,3,0.1) ; \\
& C_{5}=b_{5}=(12,5,0.1) .
\end{aligned}
$$

Figure 10 is the structural diagram of path tracking control model constructed in MATLAB/Simulink in this paper. Its running process is as follows: the current state parameters $\left(x_{c}, y_{c}, \theta_{c}\right.$, and $\left.\varphi\right)$ of the midpoint of the rear axle are obtained through a vehicle-mounted sensor. Vector quantity $\left(x_{c}, y_{c}\right)$ is input into path tracking module, through which the ideal steering angle $\varphi_{i}$ of the current reference 


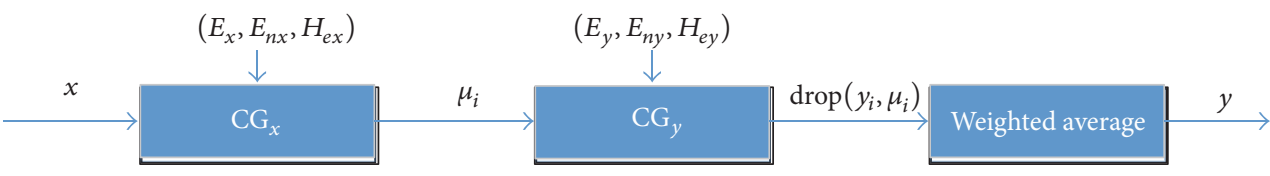

(a)

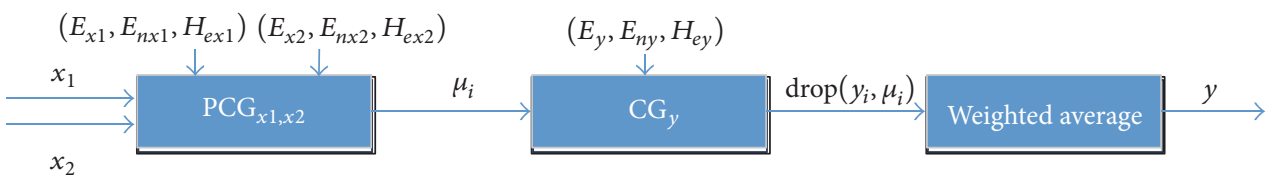

(b)

Figure 7: Single positive cloud rule generator.

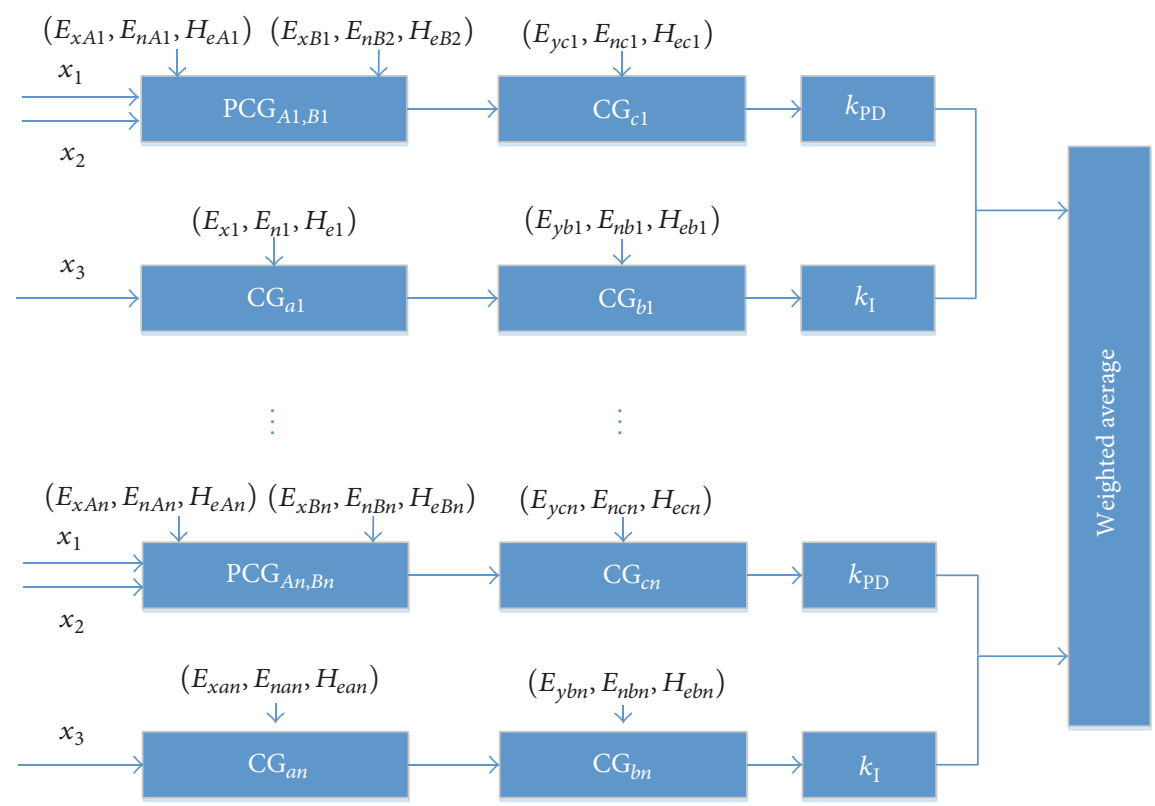

FIGURE 8: Multiple rule reasoning model.

point could be calculated. Furthermore, $\Delta \varphi$ is obtained by comparing with the actual steering angle $\varphi$ and taken as the input of multirule generator to obtain the adjustment amount of the steering angle in the parking process. With the movement of the target point in the planning path, $\Delta \varphi$ is repetitively calculated until the tracking is completed.

Parking path tracking control model is established on MATLAB/Simulink simulation platform. It is assumed that the vehicle is running at the speed of $2 \mathrm{~m} / \mathrm{s}$, and the tracking simulations on different paths are carried out, respectively.

The trajectory is assigned as $y=x$ and $x \in(0,4)$. The starting point of the path is $(0,0)$, and direction angle is $0^{\circ}$. The results of tracking simulation are shown in Figure 11, where the full line is the prescribed path and the dotted line is the tracking trajectory. Ten sample points are selected to calculate the standard deviation, which is 0.15 .

The trajectory is specified as $x^{2}+y^{2}=1$. The starting point of the path tracking is $(-2,0)$, and the direction angle is $0^{\circ}$. The simulation results are shown in Figure 12. Twenty-five sample points are selected to calculate the standard deviation, which is about 0.1 .

According to Figures 11 and 12, as for the straight line tracking, since the steering angle variation is 0 , after the rapid convergence at the starting point in tracking, the fluctuation of the tracking points is less. As for the circular trajectory tracking, the steering angle significantly changes. The tracking model can achieve rapid convergence, and the tracking error is within the reasonable range.

\section{Experiment and Result Analysis}

Real vehicle experiment of automatic parking consists of the following components: automatic parking control system, parking space scene detection equipment, WAVEBOOK512H portable dynamic data acquisition system (including a notebook computer, and DASlab8.0 data analysis software), Agilent U1620A portable oscilloscope, tape, connecting wire, and Haima Familia test vehicle. 


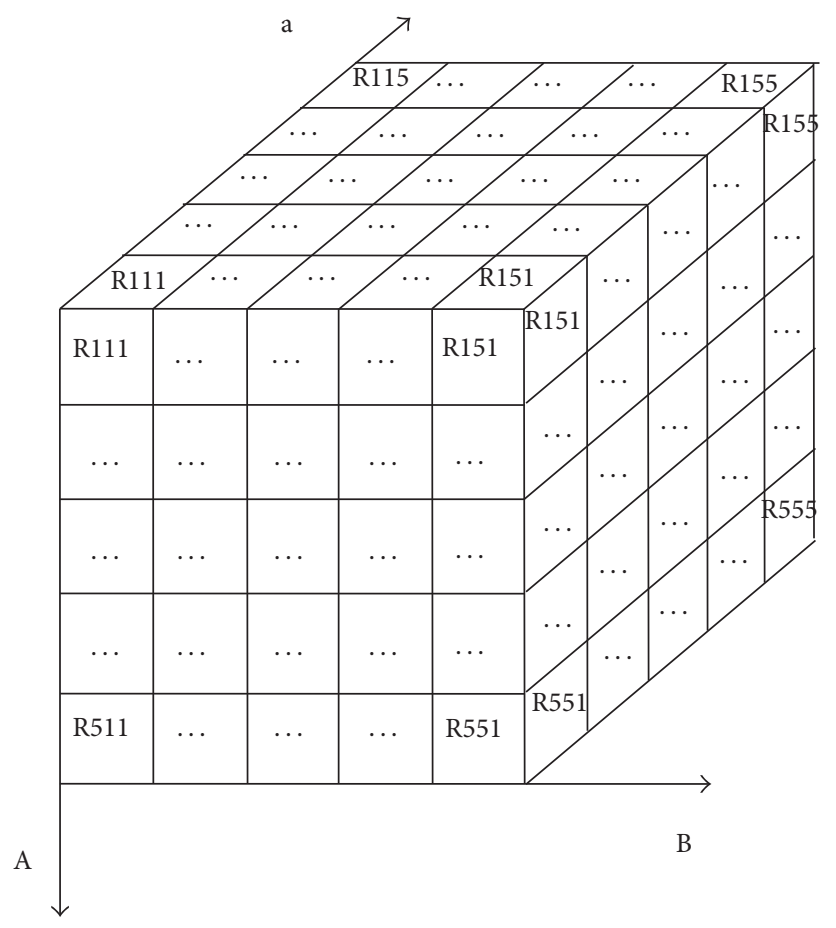

Figure 9: Rule library.

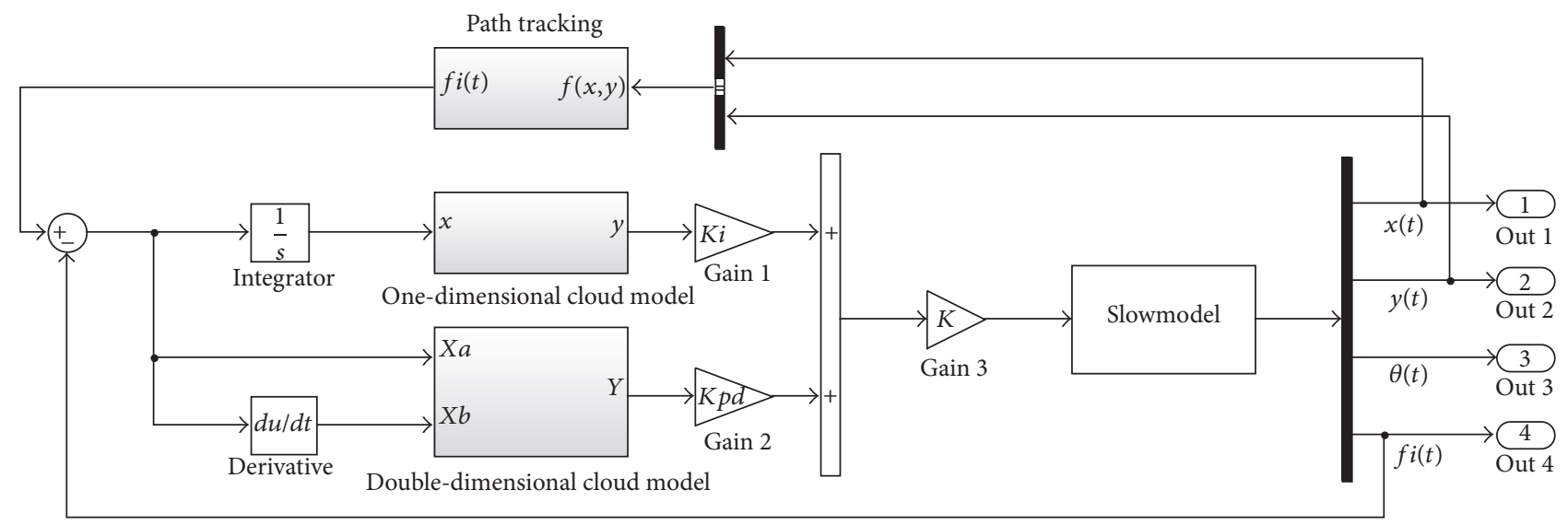

FIGURE 10: Path tracking control model.

Hardware circuit board of the automatic parking controller is developed according to the circuit principle diagram. The parking controller is loaded on the vehicle to carry out the test. Experimental diagram of parking controller hardware circuit board and parking controller loading test is shown in Figure 13.

The ultrasonic sensor of the system is mainly arranged in the front and rear, as well as the right side of the vehicle. The front and rear sensors are mainly short range radar, and the right one is mainly long distance sensor, which is shown in Figure 14.

In the parking test field, the test site is arranged according to the size of target parking space of the tested vehicle determined in the second section. The system is loaded on the tested vehicle with adaptability adjustment. The automatic parking system is started for actual parking experiment, which is shown in Figure 15.

At the starting point of the vehicle parking, the geometric method is utilized to plan the ideal parking trajectory. The segmented curve is fit to the parking path. Path tracking control model is used to track the path. The starting point is $(7.1,2.3)$ and direction angle is $0^{\circ}$, when $D_{\text {lateral }}=0.5 \mathrm{~m}$.

Three different lateral distances $\left(D_{\text {lateral }}\right)$ of the model car are planned to execute the roadside parallel autoparking experiments. Experimental results and pictures for a fixed vehicle speed of $5 \mathrm{~km} / \mathrm{s}$ are shown here for explanation. Experimental results are listed in Table 2 for comparison with the data from the PID controller and cloud model controller 
TABLE 2: Path tracking control performance comparison.

\begin{tabular}{|c|c|c|c|c|c|c|}
\hline \multirow{3}{*}{ Control scheme } & \multicolumn{6}{|c|}{ Experimental data for the following lateral distances $\left(D_{\text {lateral }}\right)$} \\
\hline & \multicolumn{2}{|c|}{$D_{\text {lateral }}, 0.5 \mathrm{~m}$} & \multicolumn{2}{|c|}{$D_{\text {lateral }}, 1.0 \mathrm{~m}$} & \multicolumn{2}{|c|}{$D_{\text {lateral }}, 1.5 \mathrm{~m}$} \\
\hline & $\begin{array}{c}\text { Minimum of } Y \\
\text { error }(\mathrm{m})\end{array}$ & $\begin{array}{c}\text { Maximum of } Y \\
\text { error }(\mathrm{m})\end{array}$ & $\begin{array}{c}\text { Minimum of } Y \\
\text { error }(\mathrm{m})\end{array}$ & $\begin{array}{c}\text { Maximum of } Y \\
\text { error }(\mathrm{m})\end{array}$ & $\begin{array}{l}\text { Minimum of } Y \\
\text { error }(\mathrm{m})\end{array}$ & $\begin{array}{c}\text { Maximum of } \\
Y \text { error }(\mathrm{m})\end{array}$ \\
\hline No control & -1.03 & -0.01 & -0.98 & -0.02 & -1.02 & -0.04 \\
\hline PID control & -0.1 & 0.2 & -0.09 & 0.15 & -0.12 & 0.18 \\
\hline $\begin{array}{l}\text { Cloud model } \\
\text { control }\end{array}$ & -0.01 & 0.06 & -0.02 & 0.08 & -0.02 & 0.1 \\
\hline
\end{tabular}

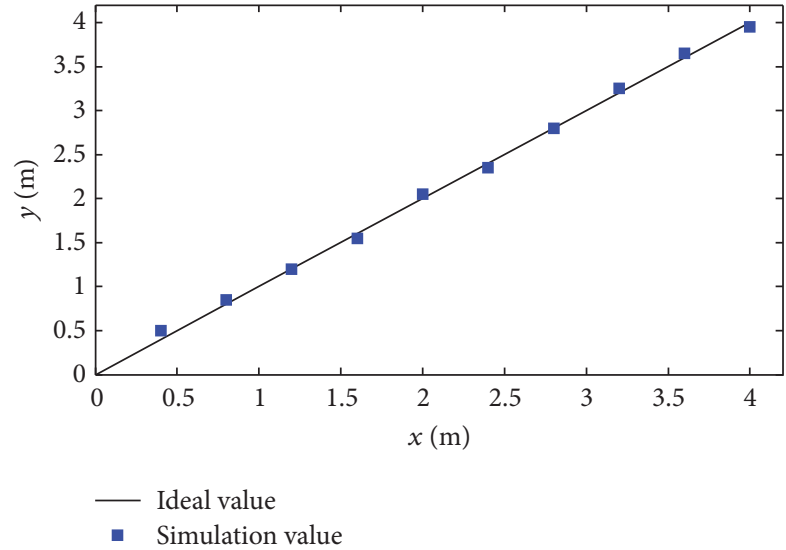

FIGURE 11: Straight path tracking.

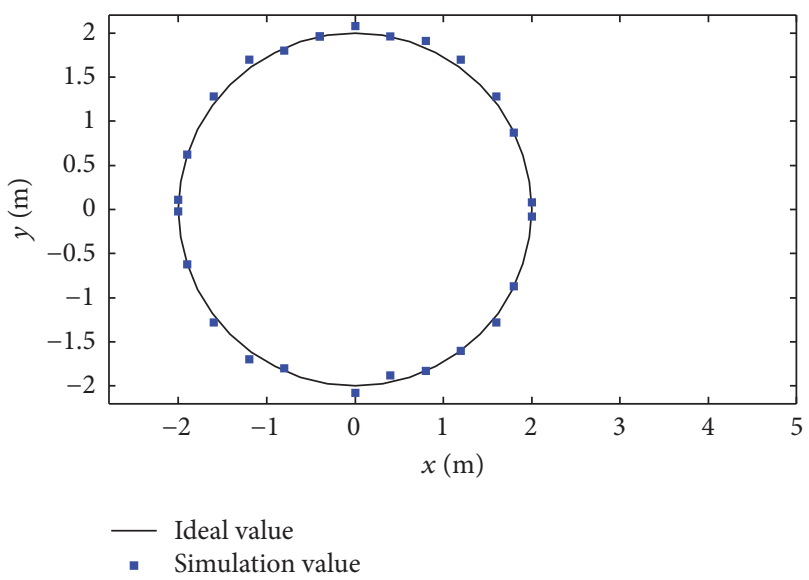

FIgURE 12: Circular path tracking.

and without controller. Four instantaneous motion pictures are shown in Figure 15.

Experimental process without controller: the wheel speed signal outputted by the wheel speed sensor in the ABS (antilock brake system) system is collected. The number of each square wave pulse signal outputted by the wheel is fixed in every circular; that is, the travelled distance (arc length) is certain. So, the number of pulses acquired can be used to calculate the distance the wheel travels (arc length). In the absence of control, there is a corresponding relationship between the distance travelled (arc length) by the wheel and the steering angle when $D_{\text {lateral }}$ is fixed. Therefore, once the arc length of each stage is reached, the microcontroller outputs a fixed steering wheel angle to achieve the parking process. At this time, the trajectory can be calculated without controller.

The trajectory of the vehicle's motion, the tracking error of minimum of $Y$ error, and the tracking error of maximum of $Y$ error are shown in Figure 16. It can be observed that the model vehicle follows the specified autoparking path backwards to the target position with a small tracking error. The minimum and maximum of $Y$ error tracking of cloud model controller are $-0.01,0.06 ;-0.02,0.08$; and $-0.02,0.1$, respectively. It can also be observed from Table 2 that the dynamic control performance of the cloud model controller is better than that of PID controller. The trajectory without controller reflects the adverse impact of time delay on automatic parking. Simultaneously, it can be observed that the cloud model controller can be better to effectively reduce the adverse impact of time delay.

Because the control parameters of the PID controller are fixed, it is impossible to adjust quickly and rationally for the uncertainty of the time delay. The situation of lag and overshoot in the control process can be found. In the case of no controller, the problem of delay will further be aggravated in the process of automatic parking path tracking. The reason is that all the time delays effect will be superimposed, resulting in increasing error and even leading to parking accident finally. However, under the cloud model controller, because of the establishment of cloud model reasoning rule base and the combination of the one-dimensional and two-dimensional cloud model mapper can solve the adverse effect of time delay and improve the robustness of the system. Furthermore, cloud model controller can guarantee the stability of the system work effectively.

\section{Conclusions}

In this paper, the adverse impact of time delay on automatic parking is analyzed. In order to solve the uncertainty of time delays, uncertain cloud reasoning model is proposed. The precision of the position of the mapping point of midpoint at the rear axle on the ground is taken as the controlled target, and the deviation between the ideal steering wheel angle and actual steering angle is taken as the input. The single-rule reasoning of multidimensional cloud model is organically integrated to design a multirule tracking control model. According to the known path, the simulation experiment 

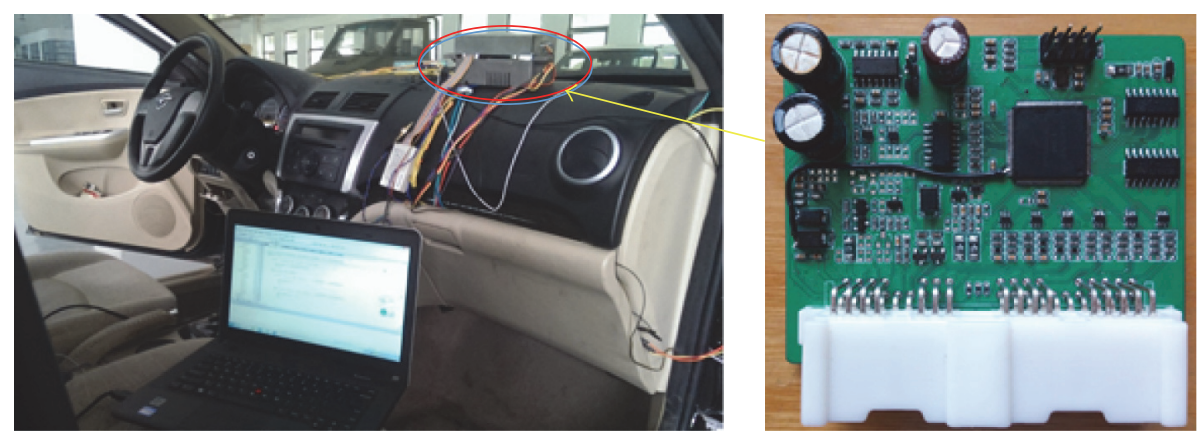

FIGURE 13: Parking controller hardware circuit board and integrated controller loading test chart.

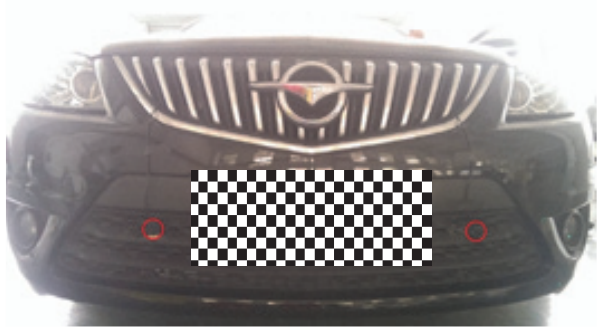

(a)

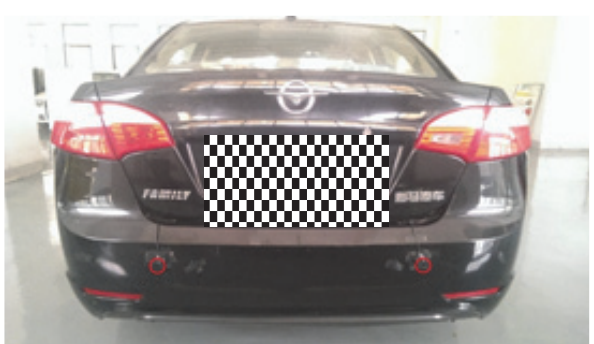

(b)

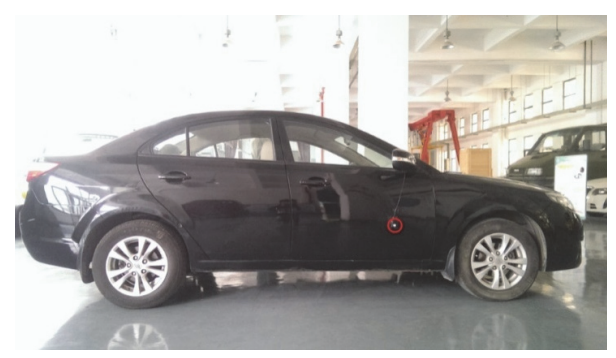

(c)

FIGURE 14: Ultrasonic sensor placement in test vehicle. (a) Front of ultrasonic radar sensor layout. (b) Rear ultrasonic radar sensor layout. (c) Side ultrasonic radar sensor layout.
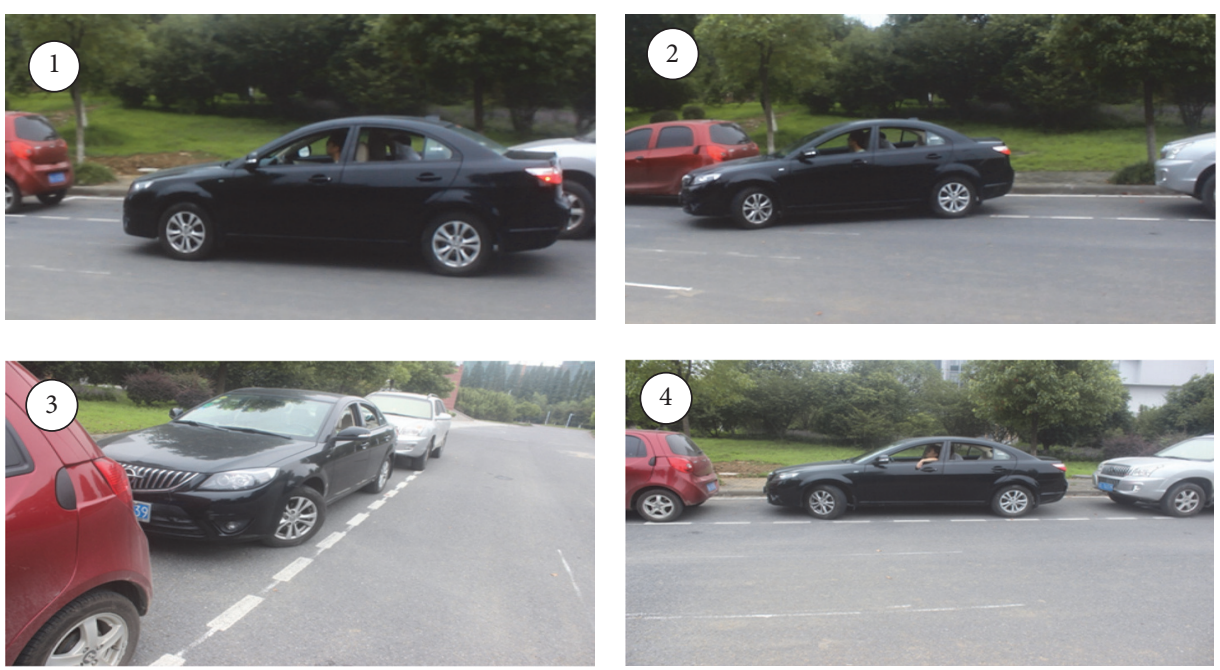

FIGURE 15: Actual test of the automatic parking system. 


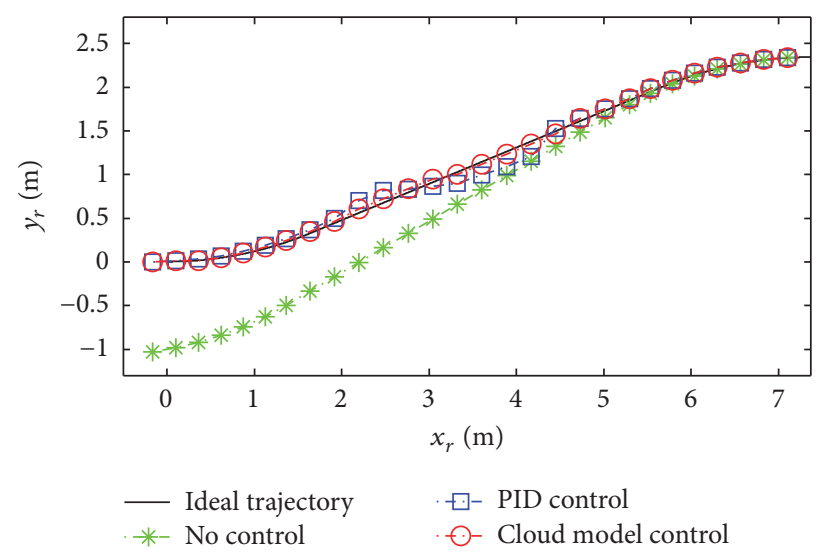

(a) $D_{\text {lateral }}=0.5 \mathrm{~m}$

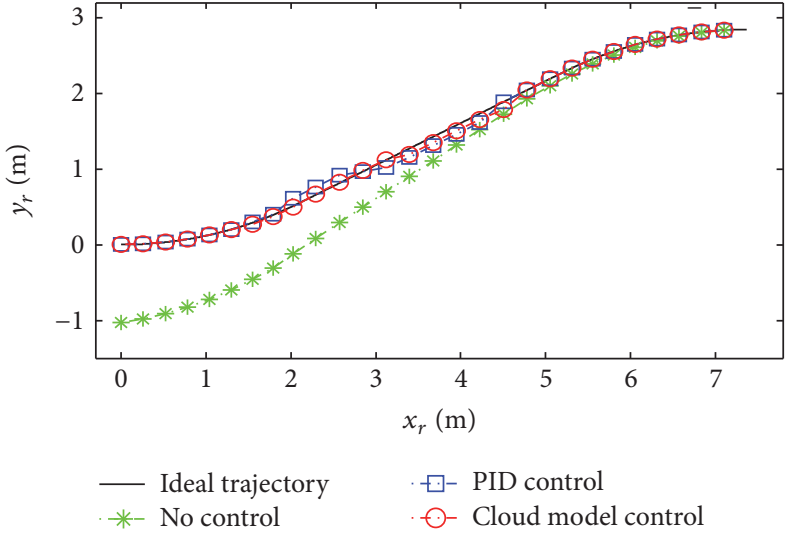

(b) $D_{\text {lateral }}=1.0 \mathrm{~m}$

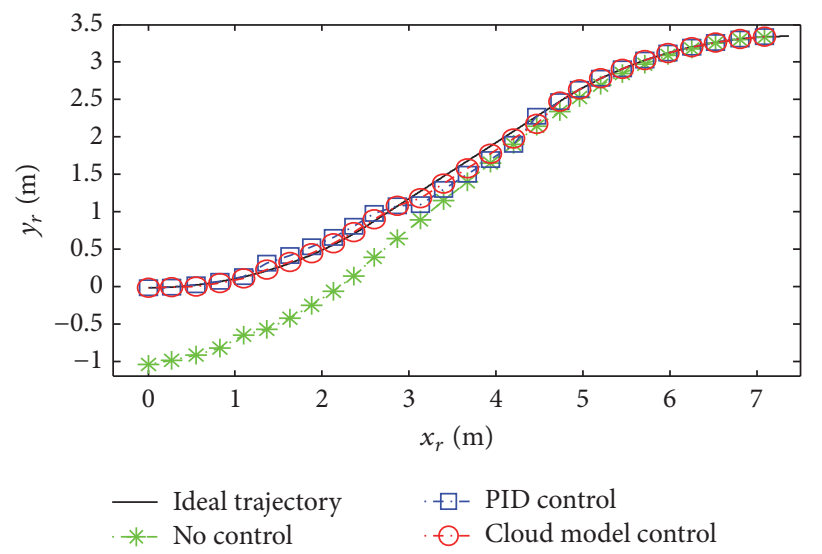

(c) $D_{\text {lateral }}=1.5 \mathrm{~m}$

FIGURE 16: The test results of path tracking.

of the parking tracking control model is carried out under constant velocity. The results show that the tracking control model has a good tracking control performance and can quickly track the specified path. The real vehicle experimental results show that cloud model controller can achieve reasonable tracking control accuracy for this automatic parking system with uncertainty of time delays. Generally, the experimental results are better than traditional PID controller. Path tracking control of automatic parking system with variable speed will be the topic of future work.

\section{Competing Interests}

The authors declare that there are no competing interests regarding the publication of this paper.

\section{Acknowledgments}

This work is financially supported by the National Natural Science Fund (nos. U1564201 and 51675235) and the CEEUSRO Innovative Capital Project in Science-Tech Bureau of Jiangsu Province (no. BY2012173).

\section{References}

[1] B. Wang, C. Shao, J. Li, D. Zhao, and M. Meng, "Investigating the interaction between the parking choice and holiday travel behavior," Advances in Mechanical Engineering, vol. 7, no. 6, pp. 1-11, 2015.

[2] M. Roca-Riu, E. Fernández, and M. Estrada, "Parking slot assignment for urban distribution: models and formulations," Omega-International Journal of Management Science, vol. 57, pp. 157-175, 2015.

[3] X. Sun, L. Chen, H. Jiang, Z. Yang, J. Chen, and W. Zhang, "High-performance control for a bearingless permanentmagnet synchronous motor using neural network inverse scheme plus internal model controllers," IEEE Transactions on Industrial Electronics, vol. 63, no. 6, pp. 3479-3488, 2016.

[4] X. Sun, L. Chen, and Z. Yang, "Overview of bearingless permanent-magnet synchronous motors," IEEE Transactions on Industrial Electronics, vol. 60, no. 12, pp. 5528-5538, 2013.

[5] X. Sun, L. Chen, Z. Yang, and H. Zhu, "Speed-sensorless vector control of a bearingless induction motor with artificial neural network inverse speed observer," IEEE/ASME Transactions on Mechatronics, vol. 18, no. 4, pp. 1357-1366, 2013.

[6] S. Yasunobu and Y. Murai, "Parking control based on predictive fuzzy control," in Proceedings of the 3rd IEEE Conference on Fuzzy Systems, pp. 1338-1341, June 1994. 
[7] M. Sugeno, T. Murofushi, T. Mori, T. Tatematsu, and J. Tanaka, "Fuzzy algorithmic control of a model car by oral instructions," Fuzzy Sets and Systems, vol. 32, no. 2, pp. 207-219, 1989.

[8] R. E. Jenkins and B. P. Yuhas, "A simplified neural network solution through problem decomposition: the case of the truck backer-upper," IEEE Transactions on Neural Networks, vol. 4, no. 4, pp. 718-720, 1993.

[9] W. A. Daxwanger and G. K. Schmidt, "Skill-based visual parking control using neural and fuzzy networks," in In Proceedings of the IEEE International Conference on Systems, Man, And Cybernetics 1995, pp. 1659-1664, ancouver, Canada, October 1995.

[10] A. Tayebi and A. Rachid, "Time-varying-based robust control for the parking problem of a wheeled mobile robot," in Proceedings of the 13th IEEE International Conference on Robotics and Automation, pp. 3099-3104, IEEE, Minneapolis, Minn, USA, April 1996.

[11] M.-C. Pai, "RBF-based discrete sliding mode control for robust tracking of uncertain time-delay systems with input nonlinearity," Complexity, vol. 21, no. 6, pp. 194-201, 2016.

[12] $\mathrm{H}$. Wu, "Decentralised robust stabilisation of uncertain largescale interconnected time-delay systems with unknown upper bounds of uncertainties," International Journal of Systems Science, vol. 47, no. 12, pp. 2816-2826, 2016.

[13] B. Gutjahr and M. Werling, "Automatic collision avoidance during parking and maneuvering - an optimal control approach," in Proceedings of the 25th IEEE Intelligent Vehicles Symposium (IV '14), pp. 636-641, Dearborn, Mich, USA, June 2014.

[14] H. Choi and B. Song, "Fault detection and handling for sensor and communication faults of an automatic valet parking system," in Proceedings of the 13th International Conference on Control, Automation and Systems (ICCAS '13), pp. 1338-1341, IEEE, Gwangju, Republic of Korea, October 2013.

[15] B. Song, D. Kim, and H. Choi, "Cooperative lateral control for automatic valet parking," in Proceedings of the 11th International Conference on Control, Automation and Systems (ICCAS '11), pp. 567-570, Gyeonggi-do, Korea, October 2011.

[16] X. Miao and L. Li, "Adaptive observer-based control for uncertain nonlinear stochastic systems with time-delay," Journal of the Franklin Institute, vol. 353, no. 14, pp. 3595-3609, 2016.

[17] Y. Batmani and H. Khaloozadeh, "On the design of suboptimal sliding manifold for a class of nonlinear uncertain time-delay systems," International Journal of Systems Science, vol. 47, no. 11, pp. 2543-2552, 2016.

[18] H. Lv, Q. Zhang, and J. Ren, "Delay-dependent $\mathrm{H} \infty$ control for a class of uncertain time-delay singular Markovian jump systems via hybrid impulsive control," International Journal of Control, Automation and Systems, vol. 14, no. 4, pp. 939-947, 2016.

[19] Q. Zhang and P.-C. Li, "Adaptive grouping chaotic cloud model shuffled frog leaping algorithm for continuous space optimization problems," Kongzhi yu Juece/Control and Decision, vol. 30, no. 5, pp. 923-928, 2015.

[20] B. Fu, D. G. Li, and M. K. Wang, "Review and prospect on research of cloud model," Application Research of Computers, vol. 28, no. 2, pp. 420-426, 2011.

[21] M.-Z. Liu, X. Zhang, M.-X. Zhang, M.-G. Ge, and J. Hu, "Rescheduling decision method of manufacturing shop based on profit-loss cloud model," Control and Decision, vol. 29, no. 8, pp. 1458-1464, 2014

[22] Y.-J. Zhang, S.-F. Shao, and N. Julius, "Cloud hypermutation particle swarm optimization algorithm based on cloud model,"
Pattern Recognition and Artificial Intelligence, vol. 24, no. 1, pp. 90-96, 2011.

[23] G.-W. Zhang, R. He, Y. Liu, D.-Y. Li, and G.-S. Chen, "Evolutionary algorithm based on cloud model," Jisuanji Xuebao/Chinese Journal of Computers, vol. 31, no. 7, pp. 1082-1090, 2008.

[24] S. Marston, Z. Li, S. Bandyopadhyay, J. Zhang, and A. Ghalsasi, "Cloud computing-The business perspective," Decision Support Systems, vol. 51, no. 1, pp. 176-189, 2011.

[25] C. Guang, B. Xiaoying, H. Xiaofei et al., "Cloud performance trend prediction by moving averages," Journal of Frontiers of Computer Science and Technology, vol. 6, no. 6, pp. 495-503, 2012.

[26] Z. Xiao, W. Song, and Q. Chen, "Dynamic resource allocation using virtual machines for cloud computing environment," IEEE Transactions on Parallel and Distributed Systems, vol. 24, no. 6, pp. 1107-1117, 2013.

[27] D. Xu, S. Yang, and H. Luo, "A fusion model for CPU load prediction in cloud computing," Journal of Networks, vol. 8, no. 11, pp. 2506-2511, 2013. 


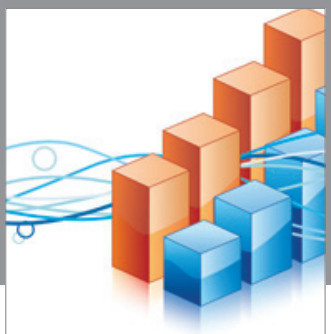

Advances in

Operations Research

vatem alat4

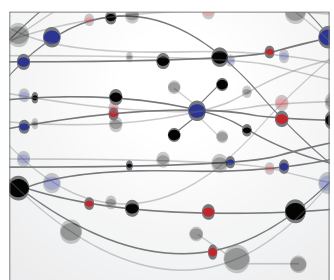

\section{The Scientific} World Journal
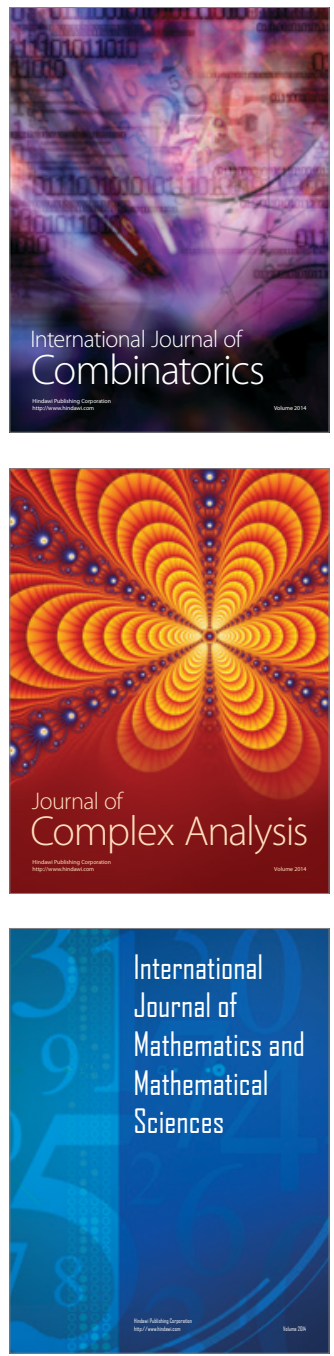
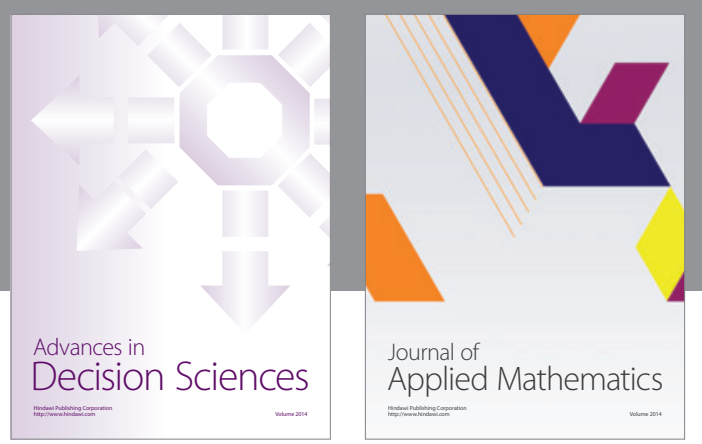

Algebra

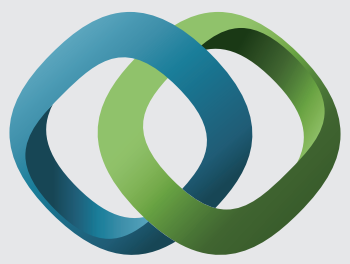

\section{Hindawi}

Submit your manuscripts at

https://www.hindawi.com
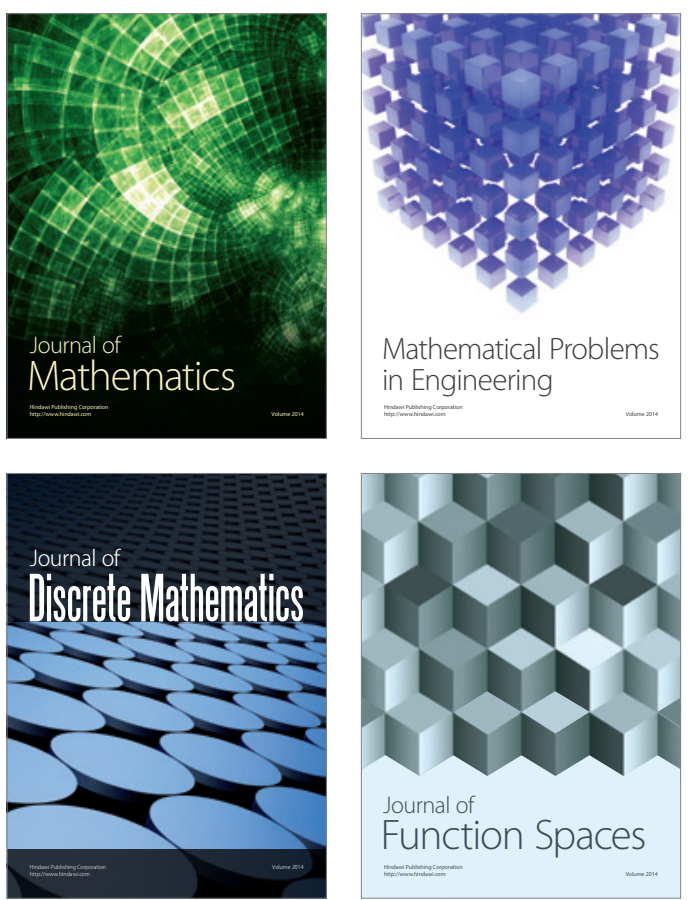

Mathematical Problems in Engineering
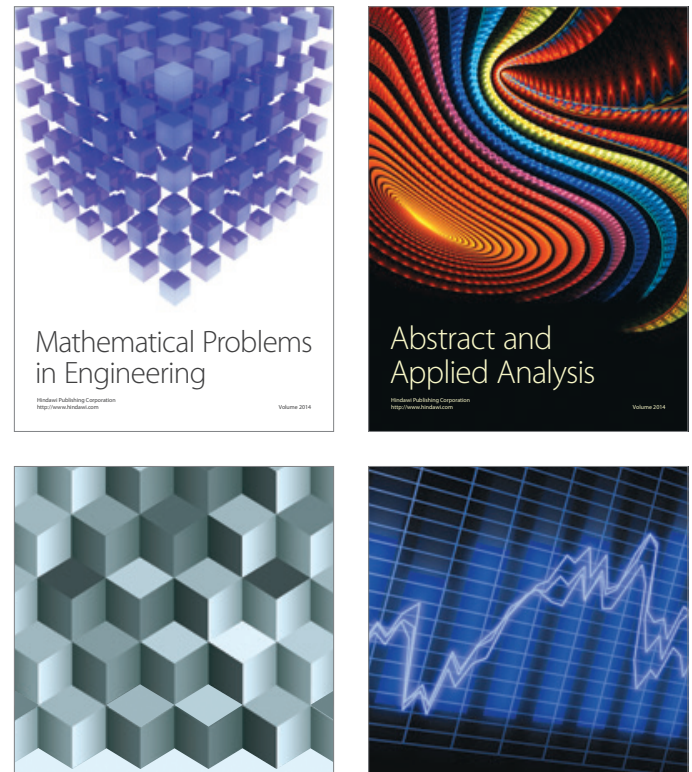

Journal of

Function Spaces

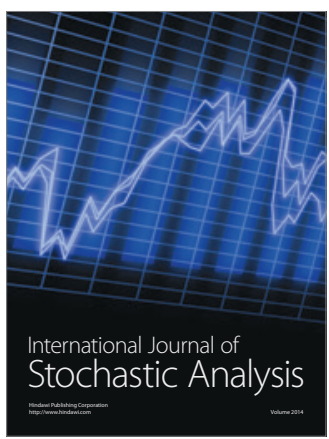

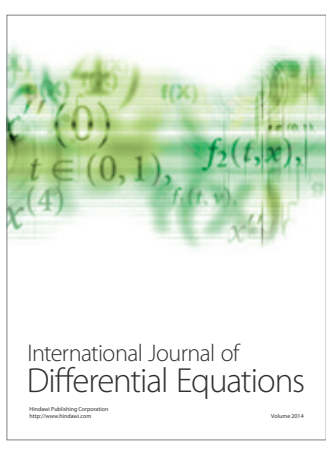
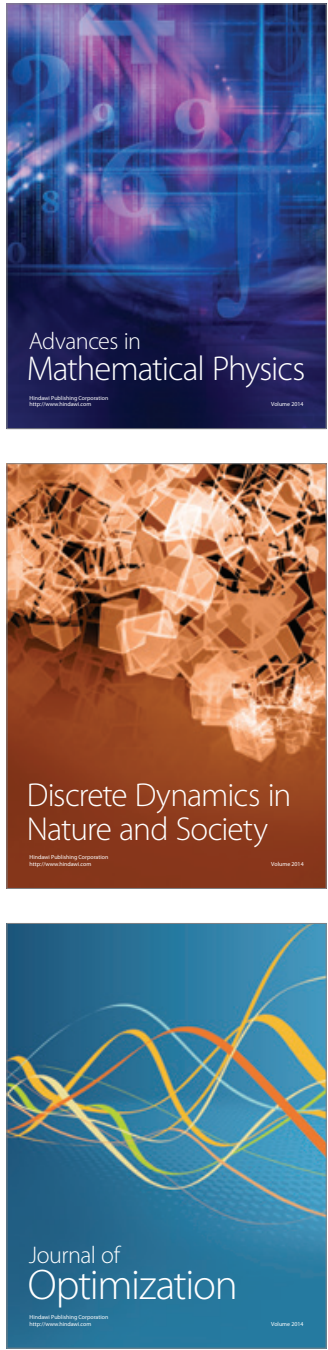